CERN-PPE/95-058

28 April 1995

\title{
Measurement of the Multiplicity of Charm Quark Pairs from Gluons in Hadronic $Z^{0}$ Decays
}

\author{
The OPAL Collaboration
}

\begin{abstract}
We have measured the multiplicity of charm quark pairs arising from gluon splitting in a sample of about 3.5 million hadronic $\mathrm{Z}^{0}$ decays. By selecting a 3 -jet event topology and tagging charmed hadrons in the lowest energy jet using leptons, we established a signature of heavy quark pair production from gluons. The average number of gluons splitting into a $c \bar{c}$ pair per hadronic event was measured to be $\overline{\mathrm{n}}_{\mathrm{g} \rightarrow \mathrm{c} \overline{\mathrm{c}}}=(2.27 \pm 0.28 \pm 0.41) \times 10^{-2}$.
\end{abstract}


R. Akers ${ }^{16}$, G. Alexander ${ }^{23}$, J. Allison ${ }^{16}$, N. Altekamp ${ }^{5}$, K. Ametewee ${ }^{25}$, K.J. Anderson ${ }^{9}$, S. Anderson ${ }^{12}$, S. Arcelli ${ }^{2}$, S. Asai ${ }^{24}$, D. Axen ${ }^{29}$, G. Azuelos ${ }^{18, a}$, A.H. Balli ${ }^{17}$ E. Barberio ${ }^{26}$, R.J.Barlow ${ }^{16}$, R. Bartoldus ${ }^{3}$, J.R.Batley ${ }^{5}$, G.Beaudoin ${ }^{18}$, S. Bethke ${ }^{14}$, A. Beck ${ }^{23}$, G.A.Beck ${ }^{13}$, C.Beeston ${ }^{16}$, T.Behnke ${ }^{27}$, K.W.Bell ${ }^{20}$, G.Bella ${ }^{23}$, S.Bentvelsen ${ }^{8}$, P. Berlich ${ }^{10}$, J. Bechtluft ${ }^{14}$, O. Biebel ${ }^{14}$, I.J. Bloodworth ${ }^{1}$, P. Bock ${ }^{11}$, H.M.Bosch ${ }^{11}$, M. Boutemeur ${ }^{18}$, S. Braibant ${ }^{12}$, P. Bright-Thomas ${ }^{25}$, R.M. Brown ${ }^{20}$, A. Buijs ${ }^{8}$, H.J. Burckhart ${ }^{8}$, R. Bürgin ${ }^{10}$, C. Burgard ${ }^{27}$, P. Capiluppi ${ }^{2}$, R.K. Carnegie ${ }^{6}$, A.A. Carter ${ }^{13}$, J.R. Carter ${ }^{5}$, C.Y.Chang ${ }^{17}$, C.Charlesworth ${ }^{6}$, D.G. Charlton ${ }^{1, b}$, S.L. Chu ${ }^{4}$, P.E.L.Clarke ${ }^{15}$, J.C.Clayton ${ }^{1}$, S.G. Clowes ${ }^{16}$, I. Cohen $^{23}$, J.E. Conboy ${ }^{15}$, O.C.Cooke ${ }^{16}$, M.Cuffiani ${ }^{2}$, S.Dado ${ }^{22}$, C.Dallapiccola ${ }^{17}$, G.M.Dallavalle ${ }^{2}$, C.Darling ${ }^{31}$, S. De Jong ${ }^{12}$, L.A.del Pozo ${ }^{8}$, H. Deng ${ }^{17}$, M.S. Dixit ${ }^{7}$, E. do Couto e Silva ${ }^{12}$, J.E. Duboscq ${ }^{8}$, E. Duchovni ${ }^{26}$, G. Duckeck ${ }^{8}$, I.P.Duerdoth ${ }^{16}$, U.C. Dunwoody ${ }^{8}$, J.E.G.Edwards ${ }^{16}$, P.G.Estabrooks ${ }^{6}$, H.G. Evans ${ }^{9}$,

F.Fabbri ${ }^{2}$, B.Fabbro ${ }^{21}$, M.Fanti ${ }^{2}$, P.Fath ${ }^{11}$, F.Fiedler ${ }^{12}$, M.Fierro ${ }^{2}$, M. Fincke-Keeler ${ }^{28}$, H.M. Fischer ${ }^{3}$, R. Folman ${ }^{26}$, D.G.Fong ${ }^{17}$, M.Foucher ${ }^{17}$, H. Fukui ${ }^{24}$, A.Fürtjes ${ }^{8}$, P. Gagnon ${ }^{6}$, A. Gaidot ${ }^{21}$, J.W. Gary ${ }^{4}$, J. Gascon ${ }^{18}$, S.M. Gascon-Shotkin ${ }^{17}$, N.I. Geddes ${ }^{20}$, C. Geich-Gimbel ${ }^{3}$, S.W. Gensler ${ }^{9}$, F.X. Gentit ${ }^{21}$, T. Geralis ${ }^{20}$, G. Giacomelli ${ }^{2}$, P. Giacomelli ${ }^{4}$, R. Giacomelli², V. Gibson ${ }^{5}$, W.R. Gibson ${ }^{13}$, J.D. Gillies ${ }^{20}$, J. Goldberg ${ }^{22}$, D.M. Gingrich ${ }^{30, a}$, M.J. Goodrick ${ }^{5}$, W. Gorn ${ }^{4}$,

C. Grandi ${ }^{2}$, E. Gross ${ }^{26}$, G.G.Hanson ${ }^{12}$, M.Hansroul ${ }^{8}$, M. Hapke ${ }^{13}$, C.K. Hargrove ${ }^{7}$, P.A.Hart ${ }^{9}$, C.Hartmann ${ }^{3}$, M.Hauschild ${ }^{8}$, C.M.Hawkes ${ }^{8}$, R.Hawkings ${ }^{8}$, R.J.Hemingway ${ }^{6}$, G. Herten ${ }^{10}$, R.D.Heuer ${ }^{8}$, J.C. Hill ${ }^{5}$, S.J. Hillier ${ }^{8}$, T.Hilse ${ }^{10}$, P.R.Hobson ${ }^{25}$, D. Hochman ${ }^{26}$, R.J.Homer ${ }^{1}$, A.K. Honma ${ }^{28, a}$, R. Howard ${ }^{29}$, R.E. Hughes-Jones ${ }^{16}$, D.E. Hutchcroft ${ }^{5}$, P. Igo-Kemenes ${ }^{11}$, D.C. Imrie ${ }^{25}$, A. Jawahery ${ }^{17}$, P.W. Jeffreys ${ }^{20}$, H. Jeremie ${ }^{18}$, M. Jimack ${ }^{1}$, A. Joly ${ }^{18}$, M. Jones ${ }^{6}$, R.W.L. Jones ${ }^{8}$, P. Jovanovic ${ }^{1}$, D. Karlen ${ }^{6}$, J. Kanzaki ${ }^{24}$, K. Kawagoe ${ }^{24}$, T. Kawamoto ${ }^{24}$, R.K.Keeler ${ }^{28}$,

R.G.Kellogg ${ }^{17}$, B.W.Kennedy ${ }^{20}$, B.J.King ${ }^{8}$, J.King ${ }^{13}$, J. Kirk ${ }^{29}$, S. Kluth ${ }^{5}$, T.Kobayashi ${ }^{24}$, M.Kobel ${ }^{10}$, D.S.Koetke ${ }^{6}$, T.P. Kokott ${ }^{3}$, S. Komamiya ${ }^{24}$, R.Kowalewski ${ }^{8}$, T.Kress ${ }^{11}$, P. Krieger ${ }^{6}$,

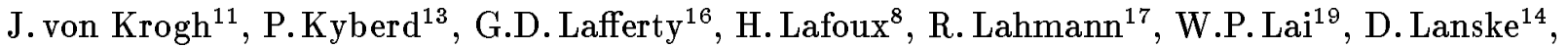

J. Lauber ${ }^{8}$, J.G. Layter ${ }^{4}$, A.M.Lee ${ }^{31}$, E. Lefebvre ${ }^{18}$, D. Lellouch ${ }^{26}$, J. Letts ${ }^{2}$, L. Levinson ${ }^{26}$, S.L. Lloyd ${ }^{13}$, F.K. Loebinger ${ }^{16}$, G.D.Long ${ }^{17}$, B. Lorazo $^{18}$, M.J.Losty ${ }^{7}$, J. Ludwig ${ }^{10}$, A. Luig ${ }^{10}$, A. Malik ${ }^{21}$, M. Mannelli ${ }^{8}$, S. Marcellini ${ }^{2}$, C.Markus ${ }^{3}$, A.J. Martin ${ }^{13}$, J.P. Martin ${ }^{18}$, T. Mashimo $^{24}$,

W.Matthews ${ }^{25}$, P.Mättig ${ }^{3}$, J.McKenna ${ }^{29}$, E.A.Mckigney ${ }^{15}$, T.J.McMahon ${ }^{1}$, A.I.McNab ${ }^{13}$, F.Meijers ${ }^{8}$, S.Menke ${ }^{3}$, F.S.Merritt ${ }^{9}$, H. Mes $^{7}$, A.Michelini ${ }^{8}$, G. Mikenberg ${ }^{26}$, D.J.Miller ${ }^{15}$, R. Mir ${ }^{26}$, W.Mohr ${ }^{10}$, A.Montanari ${ }^{2}$, T. Mori ${ }^{24}$, M.Morii ${ }^{24}$, U. Müller ${ }^{3}$, B. Nellen ${ }^{3}$, B. Nijjhar ${ }^{16}$, S.W.O'Neale ${ }^{1}$, F.G.Oakham ${ }^{7}$, F. Odorici ${ }^{2}$, H.O. Ogren ${ }^{12}$, N.J. Oldershaw ${ }^{16}$, C.J. Oram ${ }^{28, a}$, M.J. Oreglia ${ }^{9}$, S. Orito ${ }^{24}$, F.Palmonari ${ }^{2}$, J.P.Pansart ${ }^{21}$, G.N.Patrick ${ }^{20}$, M.J.Pearce ${ }^{1}$, P.D.Phillips ${ }^{16}$, J.E.Pilcher ${ }^{9}$, J.Pinfold ${ }^{30}$,

D.E.Plane ${ }^{8}$, P.Poffenberger ${ }^{28}$, B.Poli ${ }^{2}$, A.Posthaus ${ }^{3}$, T.W.Pritchard ${ }^{13}$, H.Przysiezniak ${ }^{30}$,

M.W.Redmond ${ }^{8}$, D.L. Rees ${ }^{1}$, D. Rigby ${ }^{1}$, M.G.Rison ${ }^{5}$, S.A.Robins ${ }^{13}$, N. Rodning ${ }^{30}$, J.M. Roney ${ }^{28}$,

E. $\operatorname{Ros}^{8}$, A.M.Rossi ${ }^{2}$, M.Rosvick ${ }^{28}$, P. Routenburg ${ }^{30}$, Y.Rozen ${ }^{8}$, K. Runge ${ }^{10}$, O. Runolfsson ${ }^{8}$,

D.R.Rust ${ }^{12}$, M. Sasaki ${ }^{24}$, C.Sbarra ${ }^{2}$, A.D.Schaile ${ }^{8}$, O.Schaile ${ }^{10}$, F. Scharf ${ }^{3}$, P.Scharff-Hansen ${ }^{8}$, P.Schenk ${ }^{4}$, B.Schmitt ${ }^{3}$, M.Schröder ${ }^{8}$, H.C.Schultz-Coulon ${ }^{10}$, P.Schütz ${ }^{3}$, M.Schulz ${ }^{8}$, J.Schwiening ${ }^{3}$,

W.G. Scott ${ }^{20}$, M.Settles ${ }^{12}$, T.G.Shears ${ }^{16}$, B.C.Shen ${ }^{4}$, C.H. Shepherd-Themistocleous ${ }^{7}$,

P. Sherwood ${ }^{15}$, G.P.Siroli ${ }^{2}$, A.Skillman ${ }^{15}$, A.Skuja ${ }^{17}$, A.M.Smith ${ }^{8}$, T.J. Smith ${ }^{28}$, G.A.Snow ${ }^{17}$,

R. Sobie ${ }^{28}$, S.Söldner-Rembold ${ }^{10}$, R.W.Springer ${ }^{30}$, M.Sproston ${ }^{20}$, A.Stahl ${ }^{3}$, M.Starks ${ }^{12}$,

C.Stegmann ${ }^{10}$, K.Stephens ${ }^{16}$, J. Steuerer ${ }^{28}$, B.Stockhausen ${ }^{3}$, D. Strom ${ }^{19}$, P.Szymanski ${ }^{20}$,

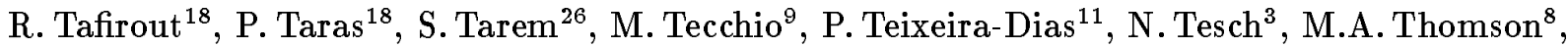

E. von Törne ${ }^{3}$, S. Towers ${ }^{6}$, M. Tscheulin ${ }^{10}$, T. Tsukamoto ${ }^{24}$, A.S. Turcot ${ }^{9}$, M.F. Turner-Watson ${ }^{8}$, P. Utzat ${ }^{11}$, R. Van Kooten ${ }^{12}$, G. Vasseur ${ }^{21}$, P. Vikas ${ }^{18}$, M. Vincter ${ }^{28}$, F. Wäckerle ${ }^{10}$, A. Wagner ${ }^{27}$, D.L. Wagner ${ }^{9}$, C.P. $\operatorname{Ward}^{5}$, D.R. Ward ${ }^{5}$, J.J. Ward $^{15}$, P.M. Watkins ${ }^{1}$, A.T. Watson ${ }^{1}$, N.K. Watson ${ }^{7}$, P. Weber ${ }^{6}$, P.S. Wells ${ }^{8}$, N. Wermes ${ }^{3}$, B.Wilkens ${ }^{10}$, G.W.Wilson ${ }^{27}$, J.A. Wilson ${ }^{1}$, T. Wlodek ${ }^{26}$, G. Wolf ${ }^{26}$, S. Wotton ${ }^{11}$, T.R. Wyatt ${ }^{16}$, G. Yekutieli ${ }^{26}$, V.Zacek ${ }^{18}$, W. Zeuner ${ }^{8}$, G.T.Zorn ${ }^{17}$. 
${ }^{1}$ School of Physics and Space Research, University of Birmingham, Birmingham B15 2TT, UK

${ }^{2}$ Dipartimento di Fisica dell' Università di Bologna and INFN, I-40126 Bologna, Italy

${ }^{3}$ Physikalisches Institut, Universität Bonn, D-53115 Bonn, Germany

${ }^{4}$ Department of Physics, University of California, Riverside CA 92521, USA

${ }^{5}$ Cavendish Laboratory, Cambridge CB3 0HE, UK

${ }^{6}$ Carleton University, Department of Physics, Colonel By Drive, Ottawa, Ontario K1S 5B6, Canada

${ }^{7}$ Centre for Research in Particle Physics, Carleton University, Ottawa, Ontario K1S 5B6, Canada

${ }^{8}$ CERN, European Organisation for Particle Physics, CH-1211 Geneva 23, Switzerland

${ }^{9}$ Enrico Fermi Institute and Department of Physics, University of Chicago, Chicago IL 60637, USA

${ }^{10}$ Fakultät für Physik, Albert Ludwigs Universität, D-79104 Freiburg, Germany

${ }^{11}$ Physikalisches Institut, Universität Heidelberg, D-69120 Heidelberg, Germany

${ }^{12}$ Indiana University, Department of Physics, Swain Hall West 117, Bloomington IN 47405, USA

${ }^{13}$ Queen Mary and Westfield College, University of London, London E1 4NS, UK

${ }^{14}$ Technische Hochschule Aachen, III Physikalisches Institut, Sommerfeldstrasse 26-28, D-52056 Aachen, Germany

${ }^{15}$ University College London, London WC1E 6BT, UK

${ }^{16}$ Department of Physics, Schuster Laboratory, The University, Manchester M13 9PL, UK

${ }^{17}$ Department of Physics, University of Maryland, College Park, MD 20742, USA

${ }^{18}$ Laboratoire de Physique Nucléaire, Université de Montréal, Montréal, Quebec H3C 3J7, Canada

${ }^{19}$ University of Oregon, Department of Physics, Eugene OR 97403, USA

${ }^{20}$ Rutherford Appleton Laboratory, Chilton, Didcot, Oxfordshire OX11 0QX, UK

${ }^{21}$ CEA, DAPNIA/SPP, CE-Saclay, F-91191 Gif-sur-Yvette, France

${ }^{22}$ Department of Physics, Technion-Israel Institute of Technology, Haifa 32000, Israel

${ }^{23}$ Department of Physics and Astronomy, Tel Aviv University, Tel Aviv 69978, Israel

${ }^{24}$ International Centre for Elementary Particle Physics and Department of Physics, University of Tokyo, Tokyo 113, and Kobe University, Kobe 657, Japan

${ }^{25}$ Brunel University, Uxbridge, Middlesex UB8 3PH, UK

${ }^{26}$ Particle Physics Department, Weizmann Institute of Science, Rehovot 76100, Israel

${ }^{27}$ Universität Hamburg/DESY, II Institut für Experimental Physik, Notkestrasse 85, D-22607 Hamburg, Germany

${ }^{28}$ University of Victoria, Department of Physics, P O Box 3055, Victoria BC V8W 3P6, Canada

${ }^{29}$ University of British Columbia, Department of Physics, Vancouver BC V6T 1Z1, Canada

${ }^{30}$ University of Alberta, Department of Physics, Edmonton AB T6G 2J1, Canada

${ }^{31}$ Duke University, Dept of Physics, Durham, NC 27708-0305, USA

${ }^{a}$ Also at TRIUMF, Vancouver, Canada V6T $2 \mathrm{~A} 3$

${ }^{b}$ Royal Society University Research Fellow 


\section{Introduction}

Heavy quark production from gluon splitting, i.e. $\mathrm{e}^{+} \mathrm{e}^{-} \rightarrow \mathrm{q} \overline{\mathrm{q} g}$, where the gluon produces two heavy quarks, $\mathrm{g} \rightarrow \mathrm{c} \overline{\mathbf{c}}$ or $\mathrm{g} \rightarrow \mathrm{b} \overline{\mathrm{b}}$, can be reliably calculated in perturbative QCD theory because it is an infrared finite quantity. However, at jet energy scales well above the heavy quark mass, as can be the case for the gluon in $\mathrm{e}^{+} \mathrm{e}^{-} \rightarrow \mathrm{Z}^{0} \rightarrow \mathrm{q} \overline{\mathrm{q}} \mathrm{g}$, large logarithmic terms arise at all orders of the perturbative expansion, spoiling its convergence. Theory predicts ( [1-3] and Table 3) values as high as 0.0033 for $\overline{\mathrm{n}}_{\mathrm{g} \rightarrow \mathrm{b}}$ and 0.0218 for $\bar{n}_{g \rightarrow c \bar{c}}$ which are the average number of $b \bar{b}$ or c $\bar{c}$ pairs produced from gluon splitting per hadronic $\mathrm{Z}^{0}$ decay, respectively. A measurement of these quantities would allow an important comparison with QCD calculation. At the moment there is only one experimental measurement for $\overline{\mathrm{n}}_{\mathrm{g} \rightarrow \mathrm{c} \overline{\mathrm{c}}}$ giving a value of $\overline{\mathrm{n}}_{\mathrm{g} \rightarrow \mathrm{c} \overline{\mathrm{c}}}=\mathbf{0 . 0 4 4} \pm \mathbf{0 . 0 1 4} \pm \mathbf{0 . 0 1 5}[4]$.

The Standard Model prediction for $\Gamma_{\mathrm{b} \overline{\mathrm{b}}} / \Gamma_{\text {had }}$ differs by $(1.8 \pm 1.3) \%$ from the experimental value [5]. If this difference were to persist when the measurements become more precise, knowledge of $\bar{n}_{g \rightarrow c \bar{c}}$ and $\bar{n}_{g \rightarrow b \bar{b}}$ can also be useful in an attempt to explain this difference as an excess of observed $b$ hadrons arising from gluon splitting into $\mathrm{b} \overline{\mathrm{b}}$.

To establish the signature of secondary charm quark pair production in hadronic decays of the $\mathrm{Z}^{0}$, i.e. $\mathrm{Z}^{0} \rightarrow \mathrm{q} \overline{\mathrm{q}} \mathrm{g}, \mathrm{g} \rightarrow \mathrm{c} \overline{\mathbf{c}}$, where $\mathrm{q}$ is any flavour quark, we looked for events with a three jet topology and assumed that the lowest energy jet was the gluon. We then looked for a lepton in the gluon jet as a heavy quark tag. While the two charm quarks might be thought of as producing two jets, we confined our search to a three jet topology by optimizing the jet finding algorithm such that most of the $\mathrm{Z}^{0} \rightarrow \mathrm{q} \overline{\mathrm{q}} \mathrm{g}, \mathrm{g} \rightarrow \mathrm{c} \overline{\mathrm{c}}$ events were identified as three jet events.

\section{The OPAL Detector}

A complete description of the OPAL detector is given elsewhere [6]. Here we describe briefly the aspects of the detector pertinent to this analysis. Tracking of charged particles is performed by a central detector, which consists of a silicon microvertex detector, a vertex detector, a jet chamber and $z$ chambers. The central detector is positioned inside a solenoid, which provides a uniform magnetic field of $0.435 \mathrm{~T}$ parallel to the beam direction. The silicon microvertex detector consists of two layers of silicon strip detectors. The inner layer covers the range $|\cos \theta|<0.83^{1}$ and the outer layer covers $|\cos \theta|<0.77$. The vertex chamber is a precision drift chamber, which covers the range $|\cos \theta|<0.95$. The jet chamber is a large volume drift chamber $4 \mathrm{~m}$ long and $3.7 \mathrm{~m}$ in diameter, providing both tracking and ionization energy loss $(\mathrm{d} E / \mathrm{d} x)$ information. The $z$ chambers measure the $z$ coordinate of tracks as they leave the jet chamber in the range $|\cos \theta|<0.72$. The coil is surrounded by a timeof-flight counter array and a lead-glass electromagnetic calorimeter with a presampler. The lead-glass covers $|\cos \theta|<0.98$. The magnet return yoke is instrumented with nine layers of streamer tubes and serves as a hadron calorimeter. Outside the hadron calorimeter are muon chambers, which cover $93 \%$ of the full solid angle.

\section{Hadronic Event Selection and Simulation}

\subsection{Hadronic Event Selection}

Hadronic $\mathrm{Z}^{0}$ decays were selected according to the number of charged tracks and the visible energy of the event, using the criteria described in Ref. [7]. Charged tracks and electromagnetic clusters unassociated with any charged track were grouped into jets using the JADE E0 recombination scheme with a $y_{\text {cut }}$ value of 0.03 [8]. The primary vertex of the event was reconstructed using the charged

\footnotetext{
${ }^{1}$ The OPAL coordinate system is defined with positive $z$ along the electron beam direction; $\theta$ and $\phi$ are the polar and azimuthal angles respectively.
} 
tracks in the event along with knowledge of the average position and effective spread of the $\mathrm{e}^{+} \mathrm{e}^{-}$ collision point.

The analysis was performed on data collected in the vicinity of the $\mathrm{Z}^{0}$ peak from 1990 to 1994 . We used the microvertex detector information for data taken after its commissioning in 1991. A total of 3.511 million hadronic events satisfied the event selection criteria.

\subsection{Event Simulation}

Monte Carlo events were used to determine the selection efficiency and background levels. For the first purpose we used the JETSET 7.4 Monte Carlo program [9] to generate $Z^{0} \rightarrow q \bar{q} g, g \rightarrow c \bar{c}$ decays in which at least one of the charm hadrons decayed semileptonically. For background studies, 2.5 million hadronic $\mathrm{Z}^{0}$ decays were also generated using the JETSET 7.3 Monte Carlo. An additional $400,000 \mathrm{Z}^{0} \rightarrow \mathrm{b} \overline{\mathrm{b}}$ events were generated. In all the above samples, the heavy quark fragmentation was parameterised using the function of Peterson et al., [10] with $\epsilon_{\mathrm{b}}=0.0057$ and $\epsilon_{\mathrm{c}}=\mathbf{0 . 0 4 6}$, in accordance with the measurements of the mean energy fraction in $\mathrm{Z}^{0} \rightarrow \mathrm{b} \overline{\mathrm{b}}$ and $\mathrm{Z}^{0} \rightarrow \mathbf{c} \overline{\mathbf{c}}$ [11]. Standard Model values of the partial widths of the $Z^{0}$ into $q \bar{q}$ were used [12]. The mixture of $c$-flavoured hadrons produced in $\mathrm{Z}^{0} \rightarrow \mathbf{c} \overline{\mathbf{c}}, \mathrm{g} \rightarrow \mathbf{c} \overline{\mathbf{c}}$ and in $\mathrm{b}$ hadron decays was as prescribed in Ref. [13]. The semileptonic branching ratios of charm hadrons and associated uncertainties are also given in Ref. [13]. All samples were processed with the OPAL detector simulation package [14]. Despite the detailed detector simulation, it was necessary to degrade the curvature, angle, and impact parameter resolution on charged tracks produced in the Monte Carlo by an additional $40 \%$ to reproduce the resolution observed in the OPAL data. This additional smearing was varied between 0 and $80 \%$ when evaluating systematic errors.

\section{Selection of $\mathrm{g} \rightarrow \mathrm{c} \overline{\mathrm{c}}$ events}

To identify the process of gluon splitting into a charm-quark pair, we made requirements on the topology of the event to select $q \bar{q} g$ events. We selected 3 -jet events, assumed the lowest energy jet as the gluon jet, and tagged a decay of a heavy quark in this jet by the presence of a prompt lepton, which can be either an electron or a muon. As the number of jets depends on the value of $y_{\text {cut }}$, we chose a value (0.03) that maximizes the number of events with three jets in a sample of Monte Carlo simulated events of the process $\mathrm{Z}^{0} \rightarrow \mathrm{q} \overline{\mathrm{q}} \mathrm{g}, \mathrm{g} \rightarrow \mathrm{c} \overline{\mathbf{c}}$ with at least one lepton coming from the decay of the charmed hadrons. Of these events $62 \%$ were found to have 3 jets, while $16 \%$ had 4 or more jets and $22 \%$ had 2 jets. About $50 \%$ of the events with three identified jets contained a lepton in the lowest energy jet coming from the charmed hadrons. We also investigated the 'Durham', 'Geneva' and the Cone jet algorithms [15], which were found to be slightly less efficient than the JADE E0 algorithm currently used. The jet energies were calculated using the relation:

$$
\mathrm{E}_{\mathrm{i}}=\mathrm{E}_{\mathrm{cm}} \frac{\sin \psi_{\mathrm{jk}}}{\sin \psi_{\mathrm{jk}}+\sin \psi_{\mathrm{ij}}+\sin \psi_{\mathrm{ik}}}
$$

where $\mathrm{E}_{\mathrm{cm}}$ is the center-of-mass energy and $\psi_{\mathrm{ij}}$ is the angle between jets $\mathrm{i}$ and $\mathrm{j}$. This equation holds for coplanar events and therefore events were rejected if the sum of the angles between the jets was smaller than $358^{\circ}$. Requiring three jets with the above selection criteria, 1.544 million events were found. We then identified the charm content of the gluon products by requiring a lepton. Only events with three jets and a lepton candidate in the lowest energy jet were selected.

Electrons were identified using an artificial neural network [16] which was trained on a sample of Monte Carlo data. Electrons from photon conversions were identified by pairing them with an oppositely charged track, and placing requirements on the pair invariant mass and vertex as in [13]. Muons were identified using information from the muon chambers in association with the drift chambers as in [17]. Electrons were required to satisfy $(2<p<6) \mathrm{GeV} / c$ and $|\cos \theta|<0.9$, while for muons, we increased the lower momentum requirement to $3 \mathrm{GeV} / c$ to suppress the background. The efficiency 
for passing these momentum cuts is approximately $32 \%$ for electrons and $20 \%$ for muons coming from the process $\mathbf{g} \rightarrow \mathbf{c} \overline{\mathbf{c}}$. For leptons satisfying these momentum cuts the tagging efficiencies are about $50 \%$. At this point the data sample contained 5761 electron events and 4186 muon events.

\subsection{Background Sources and Suppression}

We considered the following background sources:

- Jet misassignment in $\mathrm{Z}^{0} \rightarrow \mathrm{b} \overline{\mathrm{b}}$ and $\mathrm{Z}^{0} \rightarrow \mathrm{c} \overline{\mathrm{c}}$ events - cases where the lowest energy jet contains a lepton from the decay of the primary heavy quark rather than from a heavy quark in a gluon jet.

- Lepton misidentification - hadrons passing the lepton identification criteria.

- Photon conversions.

- Dalitz decays of $\pi^{0}$ and $\eta$ into a final state containing an electron.

- Decays in flight of $\pi^{ \pm}$and $\mathrm{K}^{ \pm}$into a muon.

- $\mathrm{Z}^{0} \rightarrow \mathrm{q} \overline{\mathrm{q}} \mathrm{g}, \mathrm{g} \rightarrow \mathrm{b} \overline{\mathrm{b}}$.

As the first source is from heavy flavour events only, especially $b \bar{b}$ events, while the others can be found in all $\mathrm{Z}^{0} \rightarrow \mathrm{q} \overline{\mathrm{q}}$ events, we designed cuts to reduce the $\mathrm{b} \overline{\mathrm{b}}$ contamination. Secondary vertices were sought in each jet of the event, using the algorithm described in [18], and were required to contain at least three tracks and to be separated from the primary event vertex by at least five times the error on the separation distance. The event was then rejected if any such vertex in the event contained a track with a significant impact parameter, i.e. if the impact parameter with respect to the primary event vertex $\left(d_{0}\right)$ divided by its error $\left(\sigma\left(d_{0}\right)\right)$ was greater than 2.5. This requirement reduced the overall background level by $54 \%$ (and from b $\bar{b}$ events in particular by $75 \%$ ) while retaining $61 \%$ of the $\mathrm{Z}^{0} \rightarrow \mathrm{q} \overline{\mathrm{q}} \mathbf{g}, \mathrm{g} \rightarrow \mathrm{c} \overline{\mathbf{c}}$ sample. With the addition of the above cut, the data sample contained 2754 electron events and 2061 muon events. To reduce further background from non-gluon jets, we investigated the invariant mass of the lowest energy jet. We compared Monte Carlo samples containing the process of gluon splitting into a charm quark pair with samples of $\mathrm{Z}^{0} \rightarrow \mathrm{b} \overline{\mathrm{b}}$ events in which a lepton coming from the decay of a primary $b$ quark was assigned by the jet finder to the lowest energy jet. Fig. 1 shows the distribution of the invariant mass of the lowest energy jet for these two samples. Based on the difference of the two distributions, we rejected events having their third jet invariant mass below $7.5 \mathrm{GeV}$.

After applying all the above selection criteria, we obtained 1184 events containing an electron and 930 events containing a muon. We then evaluated the remaining backgrounds in the sample as follows:

\section{Jet misassignment background}

The rate of this background was estimated from Monte Carlo simulation. Counting the number of events that passed the selection criteria in the 2.5 million hadronic $\mathrm{Z}^{0}$ decays mentioned in section 3.2 , where the lepton does not come from $\mathrm{g} \rightarrow \mathrm{c} \overline{\mathrm{c}}$ or $\mathrm{g} \rightarrow \mathrm{b} \overline{\mathrm{b}}$, and scaling to the number of hadronic events in the data sample, we estimate the background to be $382 \pm 22 \pm 44$ electron events and $249 \pm 19 \pm 26$ events in the muon channel where the first error is statistical and the second is the systematic uncertainty which is discussed in section 5.1 .

\section{Photon conversions}

Although we rejected electrons suspected of coming from photon conversion, we estimate from Monte Carlo that the number of electrons from conversions which fail to be tagged is $30.7 \pm 5.6 \%$ of the tagged 
number. Scaling the number of electrons tagged as conversions in the data by this factor, we estimate the background to be $156 \pm 28$ events. The error on this number is dominated by the uncertainty in the Monte Carlo simulation of the process, and is accounted for as systematic uncertainty.

\section{Lepton misidentification and decays in flight}

In order to estimate the background from hadrons which are erroneously identified as leptons, we used the Monte Carlo with detector simulation to estimate the probability that a charged track with a given momentum, $p$, and transverse momentum with respect to the direction of the associated jet, $p_{t}$, should be incorrectly identified as a prompt lepton. The number of background leptons in the data is derived by multiplying these fake probabilities by the number of tracks in the data. In practice this is done by deriving fake probabilities in bins of $p$ and $p_{t}$, correcting these probabilities for differences in the composition of hadronic background between the Monte Carlo simulation and the data as in [17], then weighting each data track that passes the selection criteria by the appropriate probability. These corrections carry an uncertainty of $11 \%$ for muons and $8.5 \%$ for electrons and are the main source of systematic uncertainty on the number of background events from this source. This method also accounts for the decay in flight of light hadrons into muons. The total number of background events from these sources was found to be $174 \pm 15$ in the electron channel and $506 \pm 57$ in the muon channel, where the errors are dominated by systematic uncertainties.

\section{Dalitz decays of $\pi^{0}$ and $\eta$}

The number of background events from the decay of $\pi^{0}$ and $\eta$ into $\mathrm{e}^{+} \mathrm{e}^{-} \gamma$ was estimated from Monte Carlo simulation to contribute $147 \pm 17$ electron events, where the error is predominantly statistical.

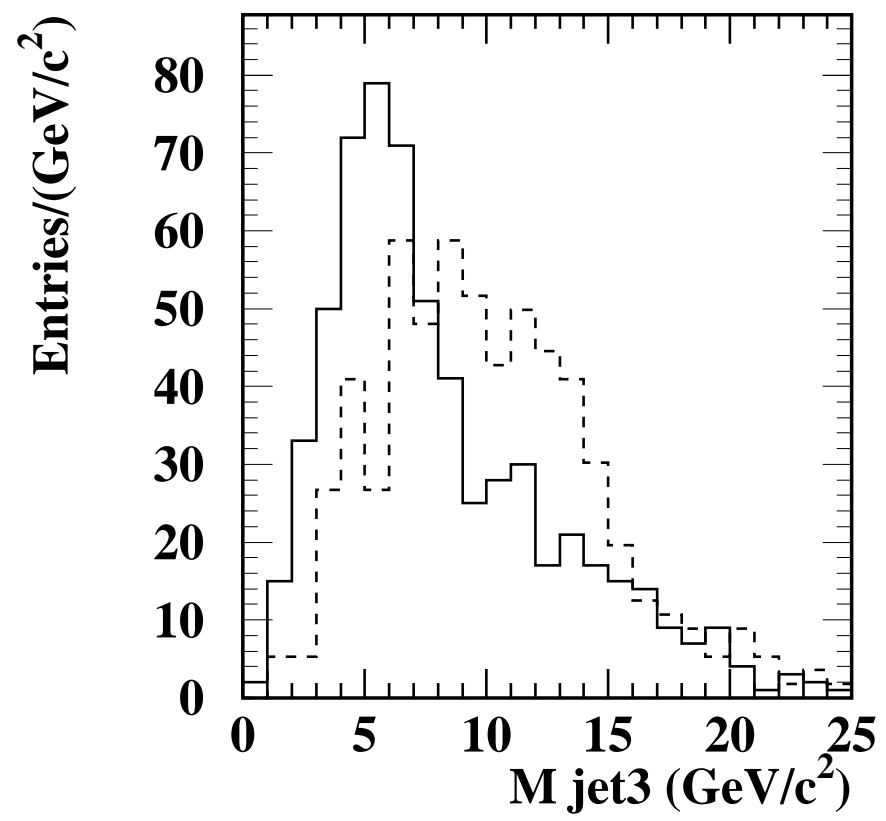

Figure 1: Invariant mass of the lowest energy jet in Monte Carlo samples of $Z^{0} \rightarrow q \bar{q} g, g \rightarrow c \bar{c}$ (dashed) and $\mathrm{Z}^{0} \rightarrow \mathrm{b} \overline{\mathrm{b}}$ (solid) for events with an identified lepton in the lowest energy jet (arbitrary normalization). 
OPAL
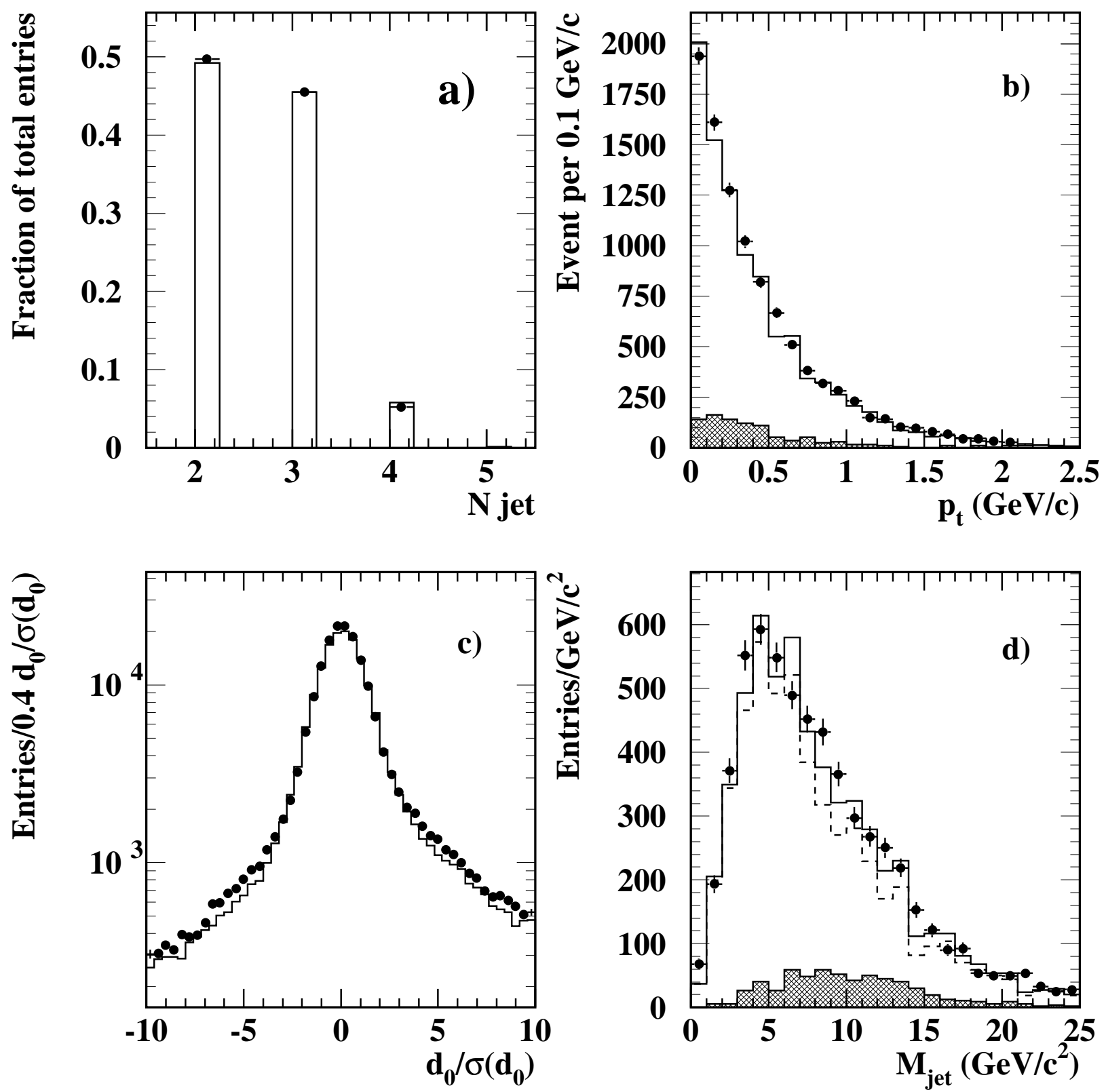

Figure 2: Comparison of Data and Monte Carlo (histograms represent the Monte Carlo and data are represented by the points). Monte Carlo figures are normalized to the number of hadronic events in the data. a) number of jets in the event, b) transverse momentum of leptons in the lowest energy jet with JETSET prediction of $\mathbf{g} \rightarrow \mathbf{c} \overline{\mathbf{c}}$ in the shaded histogram, $\mathbf{c}$ ) impact parameter significance for all tracks in events with 3 -jet topology and an identified lepton in the lowest energy jet, $d$ ) invariant mass of the lowest energy jet where the Monte Carlo without $\mathbf{g} \rightarrow \mathbf{c} \overline{\mathbf{c}}$ is the dashed histogram, the $\mathbf{g} \rightarrow \mathbf{c} \overline{\mathbf{c}}$ contribution is shaded and the solid line represents the sum of the two Monte Carlo contributions. 
$\mathrm{g} \rightarrow \mathrm{b} \overline{\mathrm{b}}$

To estimate the number of events from the process $\mathrm{Z}^{0} \rightarrow \mathrm{q} \overline{\mathrm{q} g}, \mathrm{~g} \rightarrow \mathrm{b} \overline{\mathrm{b}}$ that survive the selection criteria, we took the calculated ratio $\mathrm{R} \equiv \overline{\mathrm{n}}_{\mathrm{g} \rightarrow \mathrm{b} \overline{\mathrm{b}}} / \overline{\mathrm{n}}_{\mathrm{g} \rightarrow \mathrm{c} \overline{\mathrm{c}}}=0.132 \pm 0.047$ [4] which is based on theoretical predic-

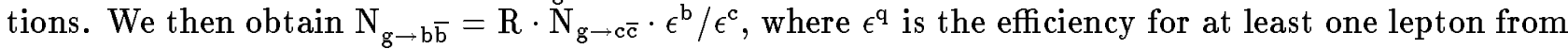
the process $g \rightarrow q \bar{q}$ to survive the selection criteria and was obtained from Monte Carlo simulation of the process and $\mathrm{N}_{\mathrm{g} \rightarrow \mathrm{Q} \overline{\mathrm{Q}}}$ is the number of events of the type $\mathrm{Z}^{0} \rightarrow \mathrm{q} \overline{\mathrm{q} g}, \mathrm{~g} \rightarrow \mathrm{Q} \overline{\mathrm{Q}}$ in the OPAL data, with $\mathrm{Q}$ being a charm or bottom quark. We estimated $\epsilon^{\mathrm{b}} / \epsilon^{\mathrm{c}}$ from Monte Carlo simulation to be $0.61 \pm 0.11$ for the electron channel and $0.45 \pm 0.15$ for the muon channel. These figures will be used in Section 5 , to account for the $\mathrm{g} \rightarrow \mathrm{b} \overline{\mathrm{b}}$ contribution in calculating $\overline{\mathrm{n}}_{\mathrm{g} \rightarrow \mathrm{c} \overline{\mathrm{c}}}$.

\subsection{Comparison Between Data and Monte Carlo}

As a major part of the background is estimated from Monte Carlo, it is of great importance to verify the compatibility of the Monte Carlo with the OPAL data. We examine the behaviour of four variables which are critical to the analysis in both the Monte Carlo simulation and the OPAL data. Fig. 2 shows these distributions: a) the number of jets in the event; $b$ ) the transverse momentum of leptons in the lowest energy jet for events with three identified jets; c) track impact parameter significance; d) invariant mass of the lowest energy jet. The small differences between Monte Carlo and data seen in c) are of little concern. As we required the number of tracks with significant impact parameter associated with any vertex to be zero, the relevant information in c) is the fraction of events passing the cut for simulated and data events. The former was found to be $41.1 \%$ while the latter is $40.2 \%$. We assigned the difference as the systematic uncertainty associated with this cut. As a final comparison we show the lepton momentum after all the selection criteria were applied (Fig. 3).

To check the jet misidentification background composition, we looked for the lepton yield in three jet events. We searched for leptons in the two highest energy jets in events that passed the selection criteria, excluding the lepton requirement in the third jet. The impact parameter cut and the third

OPAL
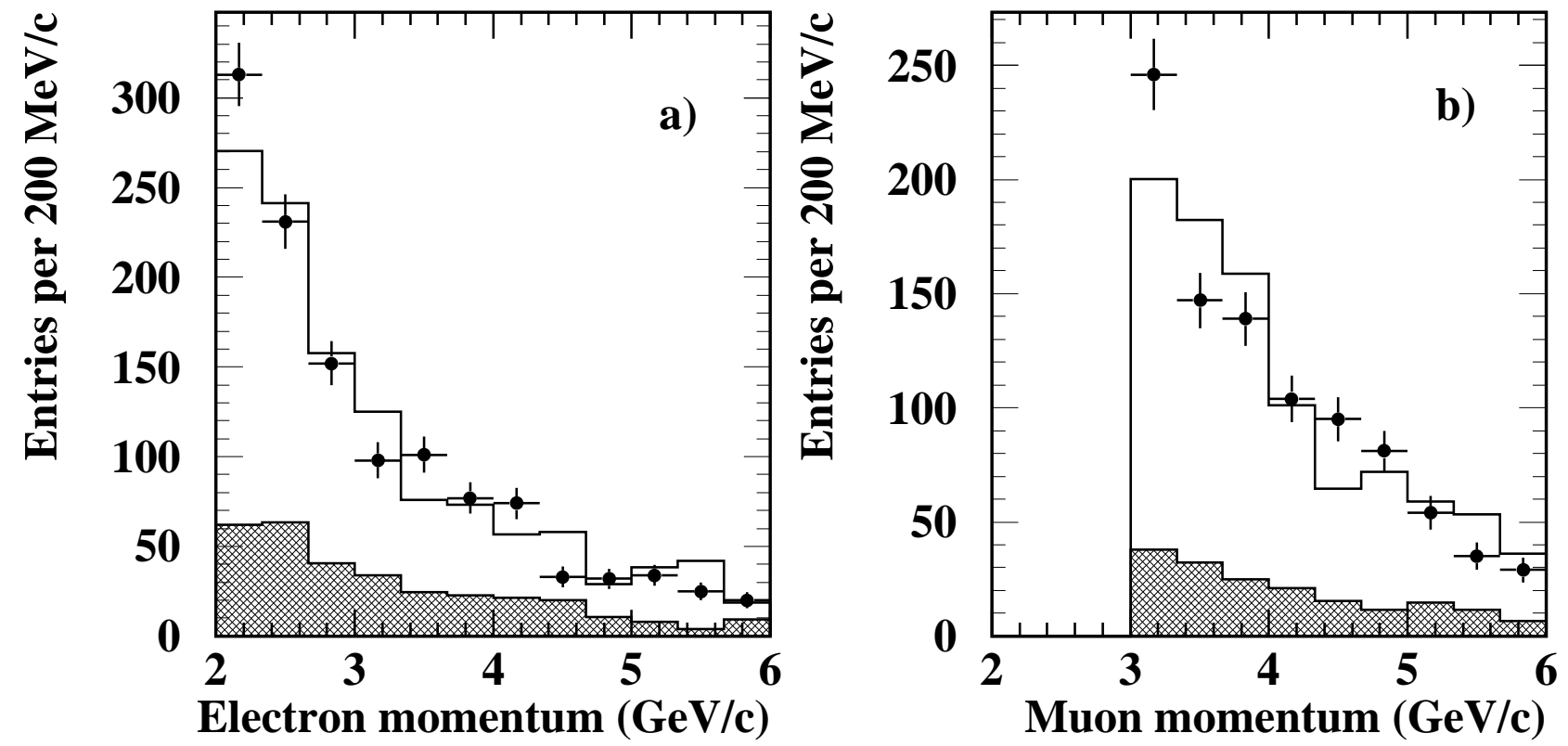

Figure 3: a) Momentum spectrum for the selected 1184 electron candidates (points) with the Monte Carlo spectrum (histograms) and the JETSET prediction for $\mathrm{g} \rightarrow \mathbf{c} \overline{\mathbf{c}}$ (shaded). $\mathrm{b}$ ) As in a) but for the 930 muon candidates. 


\begin{tabular}{|l|c|c|}
\hline Quantity & Electron channel & Muon channel \\
\hline Observed events & 1184 & 930 \\
\hline Jet misidentification & $382 \pm 22 * \pm 44$ & $249 \pm 19 * \pm 26$ \\
Residual photon conversion & $156 \pm 28$ & - \\
Lepton misidentification & $174 \pm 15$ & $506 \pm 57$ \\
Dalitz decays & $147 \pm 17 *$ & - \\
\hline Total Background & $859 \pm 28 * \pm 54$ & $755 \pm 19 * \pm 63$ \\
\hline
\end{tabular}

Table 1: Summary of measured and estimated numbers. The ' $*$ ' indicates errors which were taken as a Monte Carlo statistical uncertainty.

jet invariant mass cut were applied and leptons were identified using the same criteria as described in section 4. By requiring lepton momentum above $4 \mathrm{GeV} / c$ and transverse momentum above $1.2 \mathrm{GeV} / c$ we restricted the search to prompt leptons from $b$ hadron decay. We found the yield of such leptons per 3 -jet event to be $(2.17 \pm 0.07) \times 10^{-3}$ in the Monte Carlo and $(2.13 \pm 0.05) \times 10^{-3}$ in the data.

\section{$5 \quad$ Results and Systematic Uncertainties}

The charm quark pair multiplicity per hadronic event is related to the measured quantities by:

$$
\overline{\mathrm{n}}_{\mathrm{g} \rightarrow \mathrm{c} \overline{\mathrm{c}}}=\frac{\mathrm{N}_{\mathrm{sel}} /\left(1+\mathrm{R} \epsilon^{\mathrm{b}} / \epsilon^{\mathrm{c}}\right)}{\mathrm{N}_{\mathrm{had}} \cdot \epsilon^{\mathrm{c}} \cdot 2 \cdot \mathrm{B}(\mathrm{c} \rightarrow \mathrm{X} \ell \nu)}
$$

where $\mathrm{N}_{\text {sel }}$ is the number of events passing the selection criteria after subtraction of background events (table 1$), \mathrm{N}_{\text {had }}$ is the number of hadronic $\mathrm{Z}^{0}$ decays and $\mathrm{B}(\mathrm{c} \rightarrow \mathrm{X} \ell \nu)$ is the charm hadron semileptonic branching ratio of $(9.8 \pm 0.5) \%$ obtained by taking the average of the measurements at center of mass energies between 9.5 and $39 \mathrm{GeV}$ [19]. The relative population of weakly decaying charmed hadrons produced in the Monte Carlo for the process $\mathrm{g} \rightarrow \mathrm{c} \overline{\mathrm{c}}$ were found to be in good agreement with [19]. With $\mathrm{N}_{\text {sel }}^{e}=325 \pm 44, \mathrm{~N}_{\text {sel }}^{\mu}=175 \pm 36, \epsilon_{\mathrm{e}}^{\mathrm{c}}=(2.00 \pm 0.13) \%$ and $\epsilon_{\mu}^{\mathrm{c}}=(0.85 \pm 0.05) \%$, we obtained:

$$
\begin{aligned}
& \overline{\mathrm{n}}_{\mathrm{g} \rightarrow \mathrm{c} \overline{\mathrm{c}}}^{e}=\mathbf{0 . 0 2 1 8} \pm \mathbf{0 . 0 0 3 0} \\
& \overline{\mathrm{n}}_{\mathrm{g} \rightarrow \mathrm{c} \overline{\mathrm{c}}}^{\mu}=0.0284 \pm \mathbf{0 . 0 0 5 8}
\end{aligned}
$$

with all errors mentioned above being statistical only. Fig. 4 shows the invariant mass of the lowest energy jet for the data, after subtracting the background, and the Monte Carlo prediction normalized to the measured value of $\bar{n}_{g \rightarrow c \bar{c}}$.

\subsection{Systematic Uncertainties}

In addition to the default parameters used for the generation of the Monte Carlo samples we investigated the effects of changing the perturbative QCD model from the parton shower to second order QCD with parameters tuned as in Ref. [20]. We also varied the Peterson fragmentation input parameters $\epsilon_{\mathrm{b}}, \epsilon_{\mathrm{c}}$, the QCD running constant $\Lambda_{Q C D}$, and the Lund symmetric fragmentation parameters $a$ and $\sigma_{\mathrm{q}}$. The amount of variation in these parameters was chosen according to the range of their measured values $[11,17]$. By default heavy quark fragmentation is governed by the Peterson function in this analysis; we estimated the effect on the result of using Lund symmetric fragmentation for all quarks. Full detector simulation was not available for all these variations of the model parameters, so estimates of their effect were made by applying appropriate cuts and/or smearing to the generator output. For each of the above changes to the model, the effect on both the efficiency for selecting $\mathrm{g} \rightarrow \mathrm{c} \overline{\mathbf{c}}$ events and on the various sources of background were estimated, and their correlation taken into account in estimating the uncertainty on $\bar{n}_{g \rightarrow c \bar{c}}$. 
Another source of uncertainty arises from the modeling of the semileptonic decays of charm and bottom mesons. In addition to the default JETSET decay model, events with a prompt lepton were also reweighted as a function of the lepton momentum in the rest frame of the decaying heavy hadron to simulate different models of the semileptonic decay. The semileptonic decay model of Altarelli et al. [21] (ACCMM), with parameters tuned to CLEO data [22] for b decays and to DELCO [23] and MARK III [24] data for c decays, was used for the central values. This was combined with the $b \rightarrow D$ spectrum measured by CLEO [25] for $\mathrm{b} \rightarrow \mathrm{c} \rightarrow \ell$ decays. The model of Isgur et al. [26] (ISGW) and the modified Isgur model (ISGW ${ }^{\star \star}$ ) with the fraction of $\mathrm{D}^{\star \star}$ decays determined from CLEO data [22] were used in determining the systematic uncertainty due to the $b \rightarrow \ell^{-}$spectrum. The systematic uncertainty due to the variation of the decay model was taken into account for both the efficiency for selecting $\mathrm{g} \rightarrow \mathrm{c} \overline{\mathrm{c}}$ events and the background.

Additional sources of systematic uncertainties were considered and are described below.

- Error due to the finite size of the Monte Carlo sample used to determine the efficiencies (i.e the statistical error on the efficiencies).

- Uncertainty associated with lepton efficiencies in the Monte Carlo and due to fluctuation in the detector performances over the period of data accumulation. We assigned an uncertainty of $4 \%$ on the muon efficiency and for electrons we assigned a value of $5.5 \%$.

- Error on the number of hadrons that were misidentified as leptons. This source predominantly arises from uncertainties in the simulation of the fake probabilities in the Monte Carlo.

- Errors on the semileptonic branching ratios of charm and bottom hadrons of $5 \%$ and $8 \%$ respectively $[17,19]$.

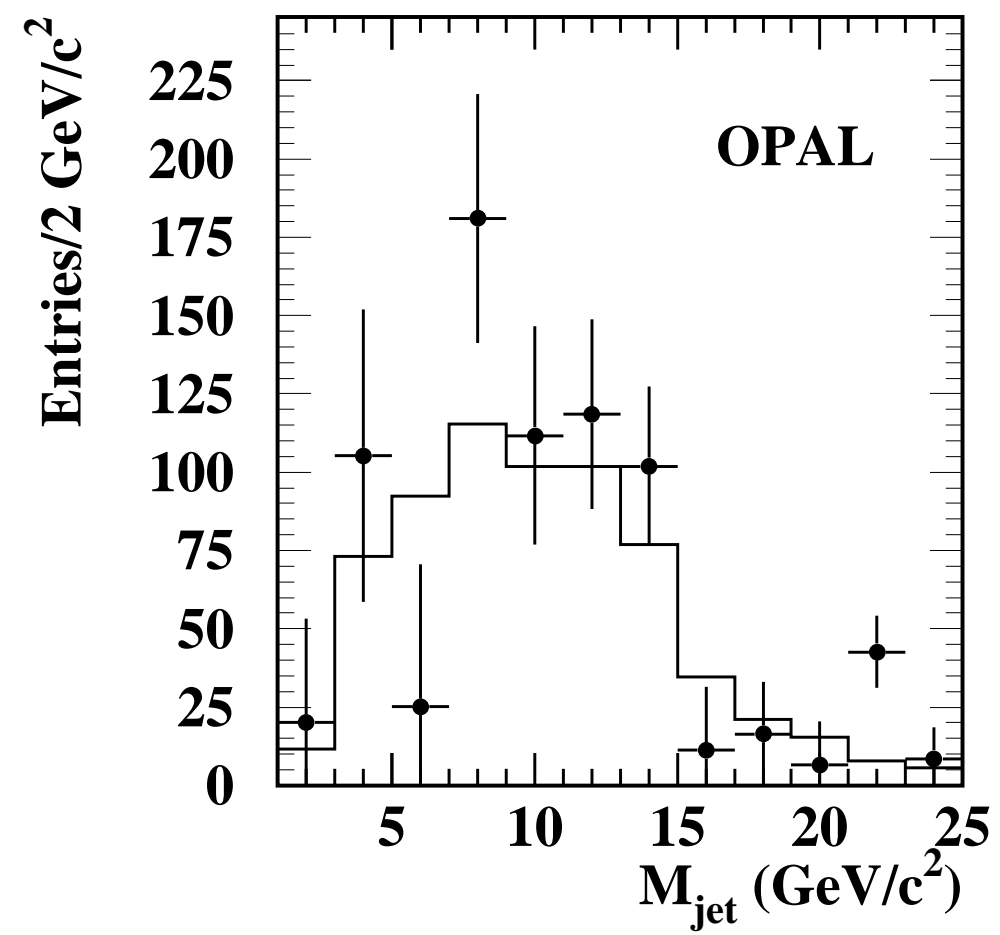

Figure 4: Invariant mass of the lowest energy jet for background subtracted data (points) and the JETSET spectrum normalized to the measured value of $\bar{n}_{g \rightarrow c \bar{c}}$ (histogram). 


\begin{tabular}{|l|c|c|}
\hline Source of uncertainty & $\delta\left(\overline{\mathrm{n}}_{\mathrm{g} \rightarrow \mathrm{c} \overline{\mathrm{c}}}^{e}\right) / \overline{\mathrm{n}}_{\mathrm{g} \rightarrow \mathrm{c} \overline{\mathrm{c}}}^{e}(\%)$ & $\delta\left(\overline{\mathrm{n}}_{\mathrm{g} \rightarrow \mathrm{c} \overline{\mathrm{c}}}^{\mu}\right) / \overline{\mathrm{n}}_{\mathrm{g} \rightarrow \mathrm{c} \overline{\mathrm{c}}}^{\mu}(\%)$ \\
\hline MC statistics & 6.3 & 6.5 \\
Lepton efficiency & 7.8 & 4.1 \\
Lepton misidentification & 4.7 & 32.6 \\
Photon conversion & 8.6 & - \\
\hline Total uncorrelated errors & 14.1 & 33.4 \\
\hline $\mathrm{O}\left(\alpha_{\mathrm{s}}^{2}\right)$ model & 1.7 & 1.3 \\
$\epsilon_{\mathrm{b}}(0.0025-0.0095)$ & 0.9 & 1.5 \\
$\epsilon_{\mathrm{c}}(0.03-0.07)$ & 1.3 & 5.1 \\
$\Lambda_{Q C D}(0.13-0.31)$ & 6.9 & 1.8 \\
Lund symmetric fragmentation parameter $a(0.13-0.32)$ & 2.3 & 1.8 \\
$\sigma_{\mathrm{q}}(0.32-0.4)$ & 2.2 & 5.2 \\
Lepton Decay model & 2.8 & 8.0 \\
\hline Total generator errors & 8.4 & 2.2 \\
\hline Track smearing $(0 \%-80 \%)$ & 1.8 & 10.1 \\
Semileptonic branching ratios and partial hadronic widths & 9.4 & 1.4 \\
\hline Vertex significance cut $(0.9 \%)$ & 1.6 & 13.1 \\
\hline Total correlated errors & 12.8 & $\mathbf{3 5 . 9}$ \\
\hline Total & $\mathbf{1 9 . 0}$ & \\
\hline
\end{tabular}

Table 2: Systematic errors. Numbers in parentheses represent the amount of variation in the source.

Table 2 lists the different sources of systematic uncertainty, their variation (where relevant) and their effect on $\bar{n}_{g \rightarrow c \bar{c}}$ for the electron and muon channels separately. We list the errors which are uncorrelated between electron and muon channels separately, for use in calculating the average result.

Averaging the two lepton channels we obtained:

$$
\overline{\mathrm{n}}_{\mathrm{g} \rightarrow \mathrm{c} \overline{\mathrm{c}}}=0.0227 \pm 0.0028 \pm 0.0041
$$

where the first error is statistical and the second is the systematic uncertainty.

The stability of the result was checked by changing the value of $y_{\text {cut }}$ between 0.01 and 0.04 . With these values we obtained $\bar{n}_{g \rightarrow c \bar{c}}=0.0211 \pm 0.0048$ and $\bar{n}_{g \rightarrow c \bar{c}}=0.0240 \pm 0.0034$ respectively (where the error is statistical only).

\section{Summary}

We have measured the charm quark pair multiplicity arising from gluon splitting by requiring a 3jet topology, selecting the lowest energy jet as the gluon jet, and tagging charmed hadrons in this jet with leptons. Our measurement is consistent with theoretical predictions and with the previous OPAL measurement, listed in Table 3 . We averaged the two OPAL measurements of $\bar{n}_{g \rightarrow c \bar{c}}$ noting that the statistical and a dominant part of the systematic uncertainties are uncorrelated obtaining $\overline{\mathrm{n}}_{\mathrm{g} \rightarrow \mathrm{c} \overline{\mathrm{c}}}=(2.38 \pm 0.48) \times 10^{-2}$.

With a ratio of $\bar{n}_{g \rightarrow b \bar{b}} / \bar{n}_{g \rightarrow c \bar{c}}=0.132 \pm 0.047$, we obtain $\bar{n}_{g \rightarrow b \bar{b}}=(3.0 \pm 1.2) \times 10^{-3}$. This value of $\bar{n}_{g \rightarrow b \bar{b}}$ is consistent with the JETSET value $\left(1.6 \times 10^{-3}\right)$ and therefore lends support to the LEP method of accounting for $\mathrm{g} \rightarrow \mathrm{b} \overline{\mathrm{b}}$ in the $\Gamma_{\mathrm{b} \bar{b}} / \Gamma_{\text {had }}$ measurements [5]. 


\begin{tabular}{|l|c|}
\hline Method & $\overline{\mathrm{n}}_{\mathrm{g} \rightarrow \mathrm{c} \overline{\mathbf{c}}}\left(\times 10^{-2}\right)$ \\
\hline Leading order calculation [2] & $\mathbf{0 . 6 0 7}$ \\
Resummed leading order calculation $[1]$ & 1.35 \\
HERWIG & $\mathbf{0 . 9 2 3}$ \\
JETSET & 1.701 \\
ARIADNE & 2.177 \\
\hline OPAL D $[4]$ & $4.4 \pm 1.4 \pm 1.5$ \\
This meas urement & $\mathbf{2 . 2 7} \pm \mathbf{0 . 2 8} \pm \mathbf{0 . 4 1}$ \\
\hline Average OPAL result & $\mathbf{2 . 3 8} \pm \mathbf{0 . 4 8}$ \\
\hline
\end{tabular}

Table 3: Theoretical predictions and experimental measurements of $\overline{\mathrm{n}}_{\mathrm{g} \rightarrow \mathrm{c} \overline{\mathrm{c}}}$. The three Monte Carlo predictions are taken from $[1]$.

\section{Acknowledgments}

It is a pleasure to thank the SL Division for the efficient operation of the LEP accelerator, the precise information on the absolute energy, and their continuing close cooperation with our experimental group. In addition to the support staff at our own institutions we are pleased to acknowledge the Department of Energy, USA, National Science Foundation, USA, Particle Physics and Astronomy Research Council, UK, Natural Sciences and Engineering Research Council, Canada, Fussefeld Foundation, Israel Ministry of Science, Israel Science Foundation, administered by the Israel Academy of Science and Humanities, Minerva Gesellschaft,

Japanese Ministry of Education, Science and Culture (the Monbusho) and a grant under the Monbusho International Science Research Program, German Israeli Bi-national Science Foundation (GIF), Direction des Sciences de la Matière du Commissariat à 1'Energie Atomique, France, Bundesministerium für Forschung und Technologie, Germany, National Research Council of Canada, A.P. Sloan Foundation and Junta Nacional de Investigacão Científica e Tecnológica, Portugal.

\section{References}

[1] M.H. Seymour, Nucl. Phys. B 436 (1995) 163.

[2] M.L. Mangano, P. Nason, Phys. Lett. B 285 (1992) 160.

[3] M.H. Seymour, Z. Phys. C 63 (1994) 99.

[4] OPAL Collab., R. Akers et al., CERN-PPE/94-217, (to appear in Z. Phys. C.).

[5] T. Behnke, D.G. Charlton, CERN-PPE/95-11, (to appear in Physica Scripta).

[6] OPAL Collab., K. Ahmet et al., Nucl. Instrum. Methods A 305 (1991) 275;

OPAL Collab., P.P. Allport et al., Nucl. Instrum. Methods A 324 (1993) 34;

OPAL Collab., P.P. Allport et al., Nucl. Instrum. Methods A 346 (1994) 476. 
[7] OPAL Collab., G. Alexander et al., Z. Phys. C 52 (1991) 175.

[8] JADE Collab., W. Bartel et al., Z. Phys. C 33 (1986) 23;

JADE Collab., S. Bethke et al., Phys. Lett. B 213 (1988) 235.

[9] T. Sjöstrand, Comp. Phys. Comm. 82 (1994) 74;

T. Sjöstrand, Comp. Phys. Comm. 39 (1986) 347;

M. Bengtsson and T. Sjöstrand, Comp. Phys. Comm. 43 (1987) 367;

T. Sjöstrand, Int. J. of Mod.. Phys. A 3 (1988) 751.

[10] C. Peterson, D. Schlatter, I. Schmitt and P.M. Zerwas, Phys. Rev. D 27 (1983) 105.

[11] OPAL Collab., G. Alexander et al., Phys. Lett. B 262 (1991) 341;

ALEPH Collab., D. Decamp et al., Phys. Lett. B 244 (1990) 551;

ALEPH Collab., D. Decamp et al., Phys. Lett. B 266 (1991) 218;

DELPHI Collab., P. Abreu et al., Z. Phys. C 56 (1992) 47;

OPAL Collab., M.Z. Akrawy et al., Z. Phys. C 47 (1990) 505.

[12] D. Bardin et al., ZFITTER, An Analytical Program for Fermion Pair Production in $e^{+} e^{-}$ Annihilation, CERN-TH.6443/92.

[13] OPAL Collab., P.D. Acton et al., Z. Phys. C 58 (1993) 523.

[14] J. Allison et al., Nucl. Instrum. Methods A 317 (1992) 47.

[15] OPAL Collab., R. Akers et al., Z. Phys. C 63 (1994) 197;

S. Bethke et al., Nucl. Phys. B 370 (1992) 310.

[16] OPAL Collab., R. Akers et al., Phys. Lett. B 327 (1994) 411.

[17] OPAL Collab., R. Akers et al., Z. Phys. C 60 (1993) 199.

[18] OPAL Collab., R. Akers et al., Z. Phys C 65 (1995) 17.

[19] CELLO Collab., H.J. Behrend et al., Z. Phys. C 19 (1983) 291;

MARKJ Collab., B. Aveda et al., Phys. Rev. Lett. 51 (1983) 443;

TASSO Collab., M. Althoff et al., Phys. Lett. B 146 (1984) 443;

TASSO Collab., M. Althoff et al., Z. Phys. C 22 (1984) 219;

TPC Collab., H. Aihara et al., Z. Phys. C 27 (1985) 39;

DELCO Collab., T. Pal et al., Phys. Rev. D 33 (1986) 2708;

JADE Collab., W. Bartel et al., Z. Phys. C 33 (1987) 339;

MARK II Collab., R.A. Ong et al., Phys. Rev. Lett. 60 (1988) 2587;

ARGUS Collab., H. Albrecht et al., Phys Lett B 278 (1992) 202.

[20] OPAL Collab., P.D. Acton et al., Z. Phys. C 58 (1993) 387.

[21] G.Altarelli et al., Nucl. Phys. B 208 (1982) 365.

[22] CLEO Collab., S. Henderson et al., Phys. Rev. D 45 (1992) 2212;

M.Worris, Thesis, Cornell University, 1991 (unpublished).

[23] DELCO Collab., W. Bacino et al., Phys. Rev. Lett. 43 (1979) 1073.

[24] MARK III Collab., R.M. Baltrusaitis et al., Phys. Rev. Lett. 54 (1985) 1976.

[25] CLEO Collab., D. Bortoletto et al., Phys. Rev. D 45 (1992) 21.

[26] N. Isgur, D. Scora, B. Grinstein and M. Wise, Phys. Rev. D39 (1989) 799. 